\title{
Phase Fluctuation Statistics
}

\author{
John B. Smyth
}

\author{
Smyth Research Associates, San Diego, Calif.
}

(Received February 14, 1964; revised March 16, 1964)

\begin{abstract}
Over the frequency range between $10^{7} \mathrm{c} / \mathrm{s}$ and $10^{10} \mathrm{c} / \mathrm{s}$, radio seeing is controlled by the distortions in the phase of the waves propagating through the earth's atmosphere. For elevation angles above two degrees, the dominant effect is refraction at $100 \mathrm{Mc} / \mathrm{s}$, the total bending of about one and one-half degrees is divided equally between the troposphere and ionosphere. Curves showing the total phase change and associated ray bending are presented for many cases of interest. Statistical fluctuations caused by instrumentation errors in measuring the physical properties of the atmosphere, and by irregularities in the medium are summarized.
\end{abstract}

\section{Introduction}

Radio fields are characterized by an amplitude and phase, both of which are, in general, functions of time and space In the idealized case of "free-space" propagation, it is possible to compute the amplitude and phase of the field everywhere in terms of the characteristics of the signal input to the radiating antenna and its transfer function. For a signal monochromatic in time, the phase of the free-space field at any point will be simply related to the phase of the signal generated. In practice, we are confronted with nonhomogeneous media ${ }^{1}$ and irregular surfaces, which provide numerous modes of propagation, few of which are amenable to direct theoretical solution in closed form. Consequently, it is difficult to predict the amplitude and phase of the radio field at points and time of interest in terms of the signal input to the terminals of the transmitting antenna. And it should be remembered that the only observable at the receiving location is the voltage output from the antenna oriented in a given direction. The situation is depicted in figure 1a. The voltage output from the receiving antenna is an explicit function of time and an implicit function of the antenna pointing direction. In fact the antenna voltage is derived from a convolution of the antenna transfer function and the spatial distribution of the radio field across its aperture. The radio field illuminating the antenna aperture will vary with time and the voltage output will be a single valued function of the aperture field; unfortunately the converse is not true.

The present paper attempts to amalgamate the data on radio phase measurements currently available, and where possible the characteristics of the phase fluctuations will be compared with proposed theoretical models. It can be shown [Smyth, 1961] that the first order effect associated with the penetration of a radio field into an inhomogeneous medium is a distortion of the phase of the field.

${ }^{1}$ For many applications the spherically stratified earth's atmosphere may be considered a nonhomogeneous medium in which are embedded a few small-scale inhomogeneities.

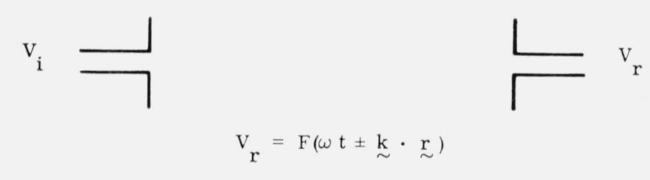

(a)

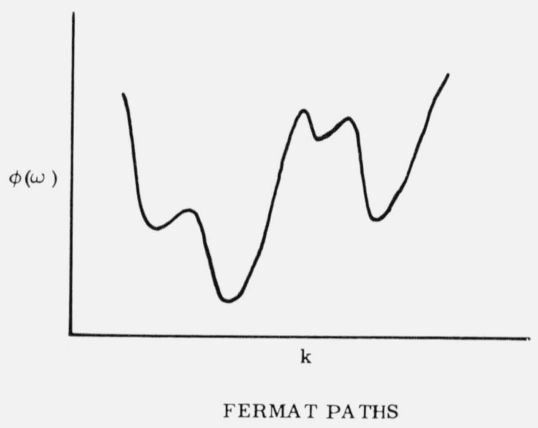

(b)

FiguRE 1. (a) Antenna voltage output as a function of time frequency and space frequency; (b) Fermat paths.

When the spatial scale of the phase distortions are larger than the radio wavelength, energy will be diffracted in the space-frequency domain. The phenomenon is quite similar to phase modulation in the time-frequency domain, where the index of modulation determines the magnitude of the energy abstracted from the main carrier for redistribution into the sidebands. At frequencies above $30 \mathrm{Mc} / \mathrm{s}$ the radio waves propagating at elevation angles greater than 2 or 3 deg will penetrate the entire atmosphere. Experience indicates that the energy will, to a first approximation, follow the geometric ray under these conditions and that second order diffraction effects result from the phase distortions. 
The radio fields propagating more nearly parallel to the earth's stratified atmosphere will suffer much larger phase perturbations which may result in an index of modulation sufficient to diffract most of the energy out of the carrier, the geometric ray, and spread it into sidebands which feed the nonoptical region. Although there is not a clear-cut distinction between these two regions, it is convenient to discuss the propagation phenomena of these two regions separately.

The argument of field quantities is always of the form $(\omega t \pm \mathbf{k} \cdot \mathbf{r})$, where $\omega / 2 \pi$ is the time frequency in cycles per second and $k / 2 \pi$ is the space frequency in cycles per unit distance. The solution to any given problem always requires a determination of the functional relationship between $k$ and $\omega$.

There are two types of phase measurement: one is the time phase between the terminals of the two antennas; the other, is the spatial variation of the phase of the field in the region of the receiving antenna. These two phase properties of the radio fields are simply related in "free space" propagation and in certain other instances, for example, waveguide transmissions. The time phase lag of the field at the receiver will depend on the Fermat path taken by the radiation between the transmitting and receiving antennas. In general there will be a dominant path and a number of secondary paths associated with the different diffraction orders created by the phase distortions. Figure $1 \mathrm{~b}$ is a schematic representation showing how the total phase between the two antenna terminals will have minima values for certain space frequencies as measured at the receiver. Within the optical region there will in general be distinct single minima associated with each space-frequency and refractive index profile. It should be remembered that this may not be the case for radio waves propagating parallel to the stratified atmosphere, multipath transmissions have been observed over short line-of-sight paths [Crawford and Jakes, 1952; DeLange, 1952].

\section{Optical Region}

Radio seeing through the earth's atmosphere is of considerable interest and importance. Almost all of the knowledge about the upper atmosphere and the total information about the universe have been obtained from an analysis of electromagnetic fields arriving at the earth's surface. The distortion of these fields by the earth's atmosphere provides information about the electromagnetic properties of this medium. To be sure, these distortions add to the problem of extracting the original information contained in the signal before it entered the atmosphere.

The macroscopic effect of the atmosphere on radio waves propagating within the optical region is to change the total time phase (travel time) and the space phase (direction of arrival). The application of Fermat's principle is basic to the solution of this part of the problem. The statistical fluctuations of the time and space phase may be obtained from the frequency of occurrence of different tropospheric and ionospheric refractive index profiles. Since the index of refraction cannot be measured precisely, the profiles should be divided into significant class intervals. Estimates of the standard deviation may be derived from a knowledge of the instrumentation errors in measuring the required physical properties of the atmosphere.

\subsection{Tropospheric Effects}

\section{a. Synoptic Refraction}

Bean, Riggs, and Horn [1959], have made a synoptic study of the vertical distribution of the radio refractive index. A description of the atmospheric profiles used in deriving the time-frequency and space-frequency variations for transmissions through the troposphere is shown in figure 2c. These consist of two exponential sections joined at an elevation of $13 \mathrm{~km}$, the upper profile being the same for all cases and represented by $N=465 \exp (-0.1568$ $h$ ) where $h$ is in kilometers and $N$ is the usual definition of refractivity. The four profiles below 13 $\mathrm{km}$ are given by: $N_{1}=220 \exp (-0.0898 h), N_{2}=280$ $\exp (-0.1177 h), N_{s}=340 \quad \exp (-0.1326 h)$, and $N_{4}=400 \exp (-0.1451 h)$. These proifles were chosen because they encompass all of the profiles which will be encountered in practice. The associated Fermat paths are represented in figure $2 \mathrm{a}$. The range of uncertainty in $N$ which would result from the uncertainties ${ }^{2}$ in measuring humidity, temperature, and pressure are about \pm 6 at $1.5 \mathrm{~km}$ and \pm 2 at $8 \mathrm{~km}$. The greatest contribution to these errors come from the errors in measuring humidity. These are only a verage values; for high temperature and relative humidity, the uncertainty in $N$ at ground level may be as large as \pm 30 .

2 International Committee for Air Navigation.

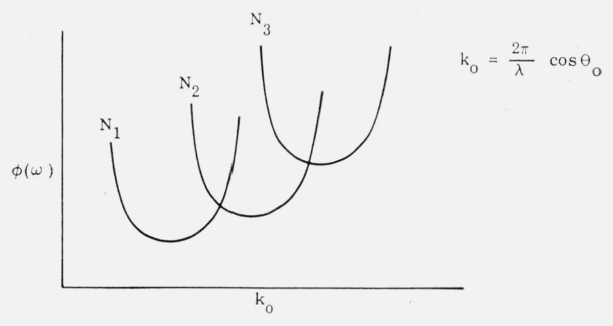

(a)
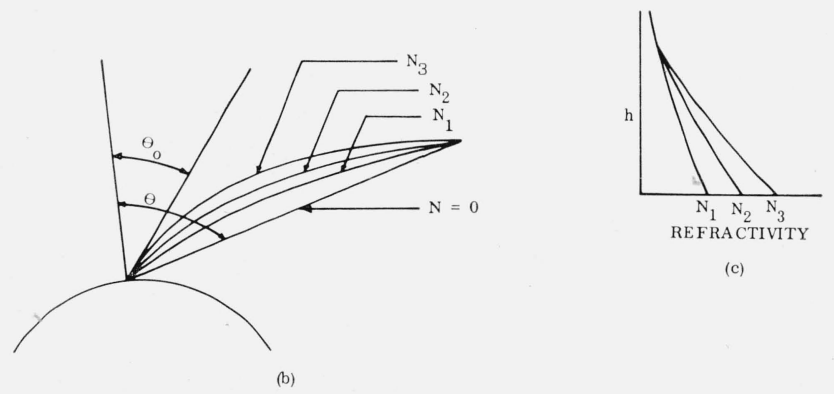

(c)

FIGURE 2. (a) Fermat paths for different index of refraction profiles; (b) ray paths through the troposphere; (c) refractive index profiles. 


\section{b. Small-Scale Inhomcgeneities}

There have been numerous theoretical studies of the propagation of radio waves through inhomogeneous media. For the case where the receiver is in view of the transmitter and the effects of the earth can be neglected, all tiers of theoretical sophistication yield identical results: the expected mean square phase fluctuation is given by

$$
\overline{(\phi)^{2}}=\overline{\left(\Delta n / n_{0}\right)^{2}} k_{0}^{2} L 1_{0},
$$

where $k_{0}$ is the space-frequency for the mean value of the index of refraction $n_{0}$, and $\Delta n$ is the mean variation of the refractive index over a distance $1_{0} . \quad L$ is the total distance traveled through the turbulent medium. This expression simply states that the expectation of the total variance of the phase over the entire path of length $L$ is just the product of the number of random variables and the individual variance, where each variable is independent with the same expectation and variance. The phase change over a distance $1_{0}$ is

$$
\Delta \int_{0}^{1_{0}} k d s=k_{0} / n_{0} \int_{0}^{1_{0}} \Delta n d s
$$

where $\Delta n$ takes on two equally probable values $+\Delta n$ and $-\Delta n$. The variance by definition is the expectation of the difference between the observed value and the mean value, squared which in this case becomes

$$
\overline{\left(\phi_{1_{0}}\right)^{2}}=\overline{\left(k_{0} / n_{0} \cdot \Delta n 1_{0}\right)^{2}} \text {. }
$$

Now in a distance $L$ there are $L / 1_{0}$ independent variables that have the same expectation and the same variance, therfore

$$
\overline{\left(\phi_{L}\right)^{2}}=L \overline{1_{0}\left(\phi_{1_{0}}\right)^{2}}
$$

which is just the original expresssion.

Figure 3 is a plot of the variation of the time phase as a function of the spatial phase that would occur at the earth's surface in the absence of the troposphere. The four $\lambda k_{0}$ curves represent observed elevation angles at the earth's surface of 0,10 , 30 , and 50 milliradian, respectively. The intersection of the appropriate $\lambda k_{0}$ contour with the applicable refractivity curve yields the total timefrequency change in passing through the entire troposphere, and the associated space-frequency

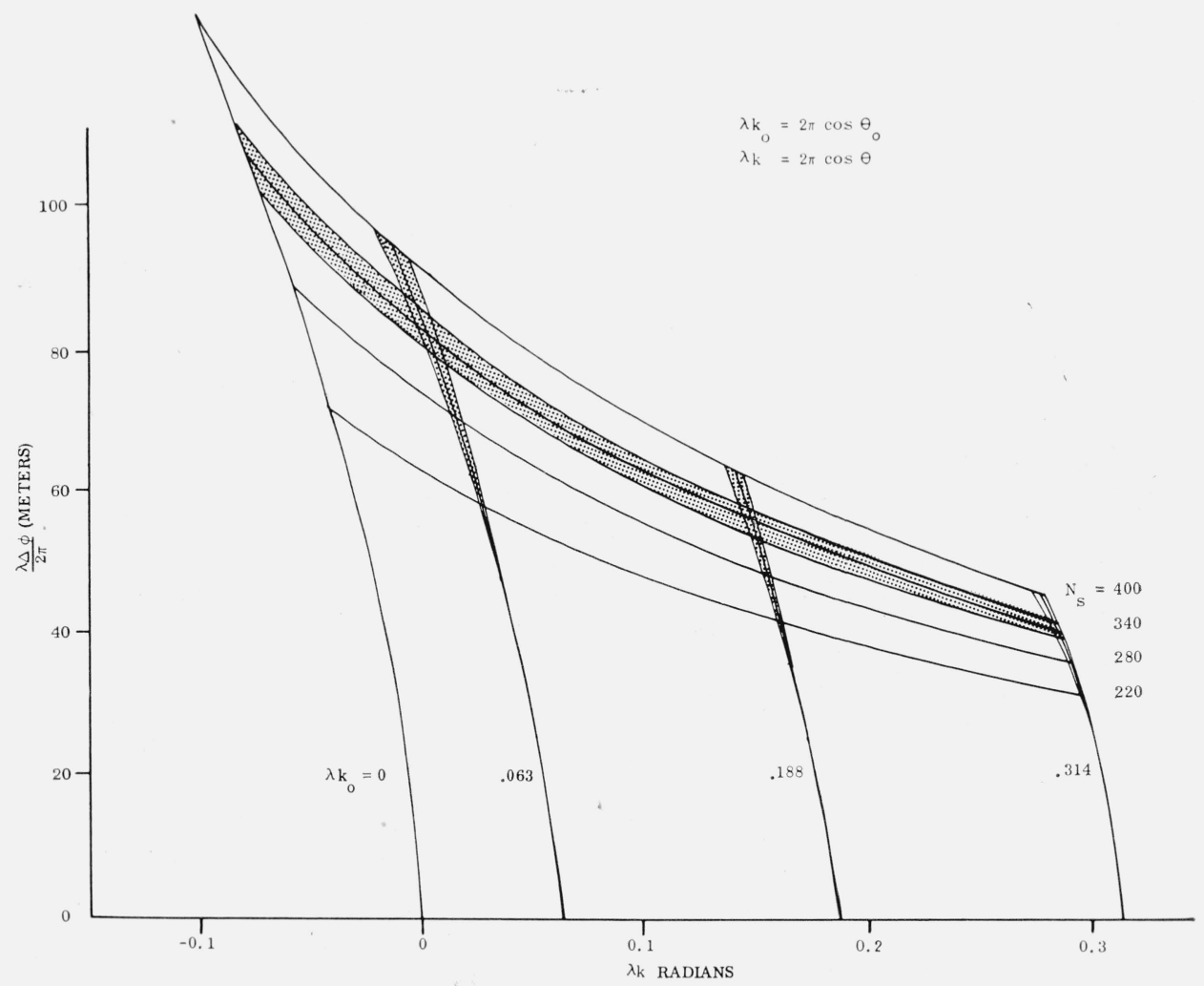

FiguRE 3. Time frequency versus space frequency for tropospheric transmission. 
change. There is an uncertainty in these values because of the errors in measuring the required physical properties of the troposphere. The stippled area represents one standard deviation. All the data which have been examined are in agreement with these curves. Thompson and Janes [1959] have measured the variations in time-frequency associated with refractivity changes over a 10 mile path. They find that the gross phase variations follow closely the changes in refractivity.

If $l_{0}$ is equal to or greater than the path length $L$, the results observed by Thompson and Janes [1959], Herbstreit and Thompson [1959]; and Deam and Fannin [1955] are easily seen to be related to the dielectric constant fluctuations associated with air mass motions. Simultaneous radio phase measurements taken by Herbstreit and Deam on $9,350 \mathrm{Mc} / \mathrm{s}$ and $1,040 \mathrm{Mc} / \mathrm{s}$ yield rms phase variations which are almost directly proportional to the frequency ratio, $12.6^{\circ}$ at $9,350 \mathrm{Mc} / \mathrm{s}$ and $1.09^{\circ}$ at $1,040 \mathrm{Mc} / \mathrm{s}$. The amplitude variation associated with these phase fluctuations was quite small, so one must agree with Deam that any postulated mechanism which constructs the field by a superposition of a large number of scatter components and the direct field is inconsistent with the observations. Herbstreit found that his observations were also in disagreement with this assumed propagation mechanism.

Thompson, Janes, and Kirkpatrick [1960a] review the atmospheric limitations on distance determination by means of radio phase measuring equipment. They compare the variance in phase of $9,400 \mathrm{Mc} / \mathrm{s}$ radio waves transmitted over a $24 \mathrm{~km}$ path with two type of corrections: first, they used the mean of the values of refractive index observed at each end of the link for the entire path; in the second instant they used the mean of five values spaced along the path. These corrections gave rms phase fluctuations of 2,600 and $1,300^{\circ}$, respectively, as compared with $3,240^{\circ}$ when no correction was made.

Booker [1958] has derived an expression for the variance in angle of arrival in terms of the variance in phase over the path,

$$
\overrightarrow{(\theta)^{2}}=2 /\left(k_{0}^{2} L^{2}\right) \overline{(\phi)^{2}} .
$$

This expression gives a standard deviation of \pm 1 milliradian for the data reported by Thompson. This is of the same magnitude as the curves of figure 3. Aarons and Castelli [1958] measured refraction and scintillation of $3.2 \mathrm{~cm}, 0.87 \mathrm{~cm}$, and $218 \mathrm{Mc} / \mathrm{s}$ solar radiation. Refraction at $0^{\circ}$ elevation angle ranged between $1^{\circ} 35^{\prime}$ and $45^{\prime}$, with an average of $1^{\circ} 05^{\prime}$. The magnitude of the scintillations observed indicate angle of arrival variations similar to those quoted above.

\subsection{Ionospheric Effects}

\section{a. Macroscopic Refraction}

Measurements of the electron density in the ionosphere indicate that the region of interest for computing ray bending is from $100 \mathrm{~km}$ to $1,000 \mathrm{~km}$. It will be assumed that the medium is spherically stratified and that the profile consist of a parabolic distribution below $300 \mathrm{~km}$, joined on to a hyperbolicsecant distribution above. About three-fourths of the electrons will be contained in the region above $300 \mathrm{~km}$. A ray leaving tangent to the earth will arrive at about a $10^{\circ}$ grazing angle with respect to the ionosphere, consequently, the refraction will be a rather slowly varying function of elevation angle.

Figure 4 is a time frequency - space frequency relationship for $100 \mathrm{Mc} / \mathrm{s}$ transmission through the ionosphere at Inverness, Scotland. The data were computed from ionospheric soundings at that location, applied to radiation launched at an elevation angle of zero degrees with the earth's surface and propagating to a height of 1,000 miles. It is interesting to note that all of the data-diurnal, seasonal, and for different years fit quite well to a single line. The large diurnal variation in December 1956 is particularly noteworthy. At $100 \mathrm{Mc} / \mathrm{s}$ the standard deviation in phase path is equivalent to about $150 \mathrm{~m}$.

\section{b. Small-Scale Irregularities}

Ionospheric irregularities and their movements have been the subject of considerable experimental study and theoretical speculation. Two conferences on the ionosphere sponsored by the Physical Society have been convened during the past decade, and the published proceedings of these two meetings contain a survey of the important observations made in many parts of the world. Both radio star observations and recorded transmissions from satellites have been used to deduce the height and horizontal extent of the irregularities.

Lawrence, Jespersen, and Lamb [1961] have observed the amplitude and angular scintillations of the radio star Cygnus A at Boulder, Colo. At an elevation angle of $15 \mathrm{deg}$ they observed an rms angular scintillation of $0.03^{\circ}$ at $108 \mathrm{Mc} / \mathrm{s}$ and $0.12^{\circ}$ at $53 \mathrm{Mc} / \mathrm{s}$. This agrees very well with the above relationship. At this elevation angle the total refraction of the $53 \mathrm{Mc} / \mathrm{s}$ radiation was observed to be 0.5 deg. Flood [1963] in a study of radio fadeouts has arrived at the following values of rms angular scintillation: $50 \mathrm{Mc} / \mathrm{s}, 360$ milliradians; $150 \mathrm{Mc} / \mathrm{s}, 40$ milliradians; $300 \mathrm{Mc} / \mathrm{s}, 10$ milliradians. It would appear that these values were taken off a wavelength plot.

\section{References}

Aarons, J., and J. P. Castelli (1958), Radio astronomy measurements at VHF and microwaves, Proc. IRE 46, $325-333$.

Bean, B. R., L. P. Riggs, and J. D. Horn (1959), Synoptic study of the vertical distribution of the refractive index, J. Res. NBS 63D (Radio Prop.), No. 2, 249-254.

Booker, H. G. (1958), The use of radio stars to study irregular refraction of radio waves in the ionosphere, Proc. IRE 46, $298-314$.

Crawford, A. B., and W. C. Jakes, Jr. (1952), Selective fading of microwaves, Bell System Tech. J. 31, 68-90. 


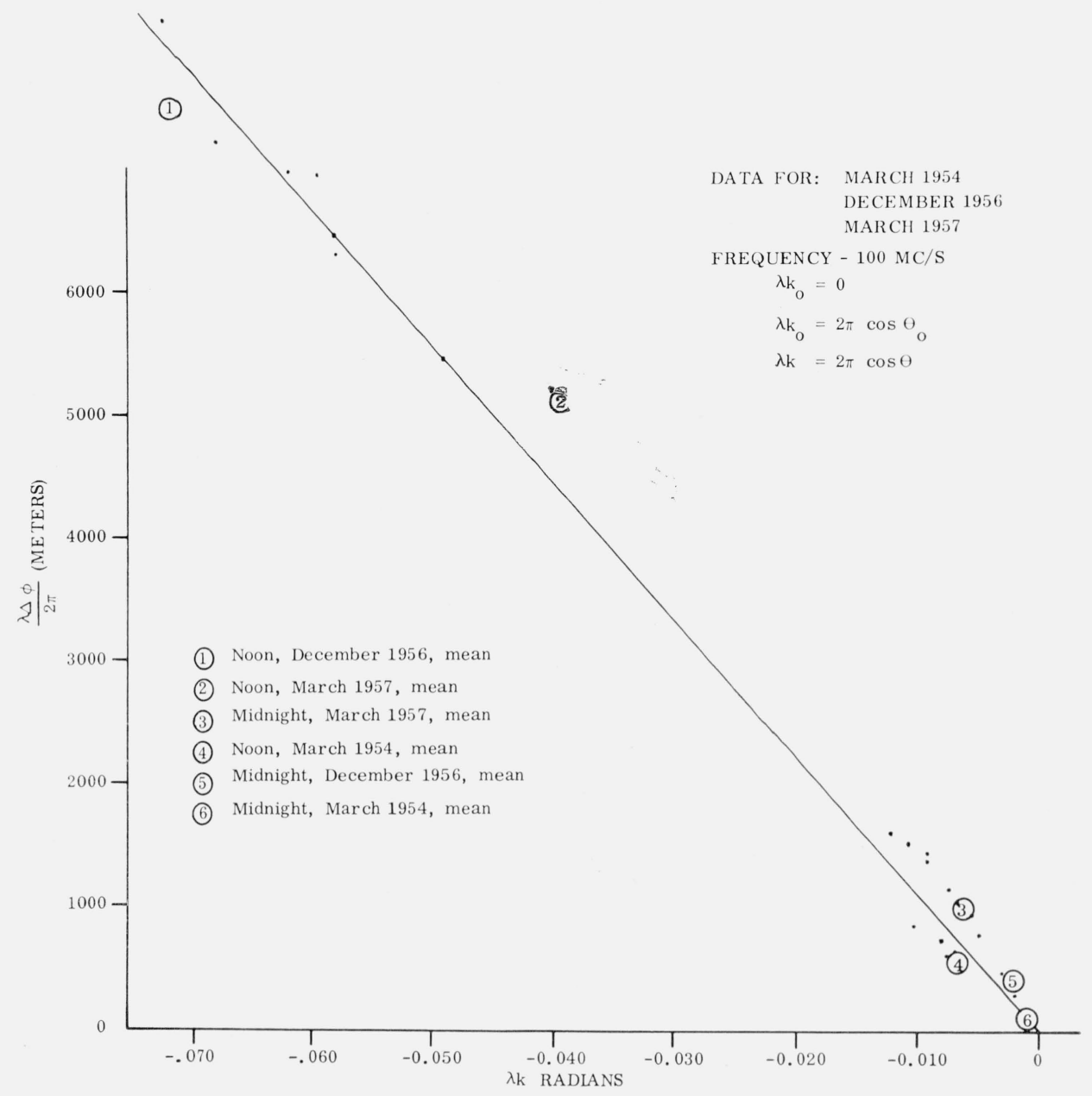

FIGURE 4. Time frequency versus space frequency for ionospheric transmissions.

Deam, A. P., and B. M. Fannin (1955), Phase difference variations in $9,350 \mathrm{Mc} / \mathrm{s}$ radio signals arriving at spaced antennas, Proc. IRE 43, 1402-1404.

DeLange, O. E. (1952), Propagation studies at microwave frequencies by means of short pulses, Bell System Tech. J. 31, 91-103.

Flood, Waiter A. (1963), A study of radio star fadeouts and their application to radar resolution, J. Geophys. Res. 68, 4129-4140.

Herbstreit, J. W., and M. C. Thompson, Jr. (1955), Measurement of the phase of radio waves received over transmission paths with electrical lengths varying as a result of atmospheric turbulence, Proc. IRE 43, 1391-1401.

Lawrence, R. S., J. L. Jespersen, and R. C. Lamb (1961), Amplitude and angular scintillations of the radio source Cygnus-A observed at Boulder, Colo. J. Res. NBS 65D, (Radio Prop.), No. 4, 333-350.

Smyth, J. B. (1961), Space analysis of radio signals, J. Res. NBS 65D (Radio Prop.), No. 3, 293-297.
Thompson, M. C. Jr., and H. B. Janes (1959), Measurement of phase stability over a low-level tropospheric path, J. Res. NBS 63D (Radio Prop.), No. 1, 45-51.

Thompson, J. C. Jr., H. B. Janes, and A. W. Kirkpatrick (1960a), An analysis of time variations in tropospheric refractive index and apparent radio path length, J. Geophys. Res. 65, 193-201.

\section{Additional Related Reference}

Thompson, M. C. Jr., H. B. Janes, and F. E. Freethey (1960b), Atmospheric limitations on electronic distance measuring equipment, J. Geophys. Res. 65, 389-393.

(Paper 68D9-397) 